\title{
Expressway Guardrail Extraction and Classification Based on Slice Method
}

\author{
Ming Huang, ${ }^{1}$ Chuanli Zhou, ${ }^{1}$ Pengpeng Huo, ${ }^{*}$ and Zhiqun $\mathrm{Wu}^{2}$ \\ ${ }^{1}$ Engineering Research Center of Representative Building and Architectural Heritage Database, \\ Ministry of Education, Key Laboratory for Urban Geomatics of National Administration of Surveying, \\ Mapping and Geoinformation, Beijing University of Civil Engineering and Architecture, Beijing 100044, China \\ ${ }^{2}$ Beijing Beijianda Technology Co., Ltd., Beijing 100044, China
}

(Received September 27, 2020; accepted November 16, 2020)

Keywords: expressway, point cloud, guardrail recognition, type distinguishment

Guardrails are an important ancillary facility of expressways. Accurate and effective identification of guardrails from road scenes plays an important role in high-precision map construction, automatic driving, road maintenance, and so on. At present, there are few research methods on guardrail recognition in highway scenes, and the existing methods have some defects such as low recognition accuracy and inability to distinguish guardrail types. In this paper, a point cloud of an expressway scene obtained by mobile laser scanning is taken as the research object. On the basis of the binary coding voxelization segmentation method, the clustering slicing method is used to recognize the guardrail target in the expressway scene according to the characteristics of the guardrail. In addition, a method for distinguishing the type of guardrail based on its cross-section characteristics is proposed to distinguish steel and concrete guardrails in a highway. The experimental results show that the proposed method can effectively identify the guardrail target in the point cloud of an expressway scene, and has high efficiency and accuracy.

\section{Introduction}

As a major ancillary facility of expressways, guardrails play an important role in preventing vehicles from crossing the road, protecting vehicles and drivers, and keeping objects off the road. In road maintenance, it is usually necessary to check the integrity and rationality of guardrails. In the case of automatic driving, vehicles usually need to detect the guardrail to prevent collision. In a high-precision map, guardrails can provide boundary information. There are two types of guardrail on both sides of the highway. One is a continuous structure with steel guardrail plates spliced together and supported by columns, which is called a steel guardrail in this paper, as shown in Fig. 1(a). The other is a concrete guardrail, as shown in Fig. 1(b). In some road sections, these two types of guardrail are connected, as shown in Fig. 1(c). Accurate and efficient extraction of guardrails from road scenes and accurate differentiation play an important role in high-precision map construction and road condition investigation.

*Corresponding author: e-mail: 2208521518001@stu.bucea.edu.cn

https://doi.org/10.18494/SAM.2020.3118 

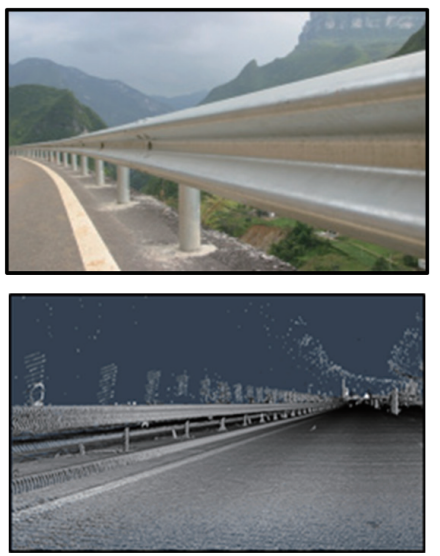

(a)
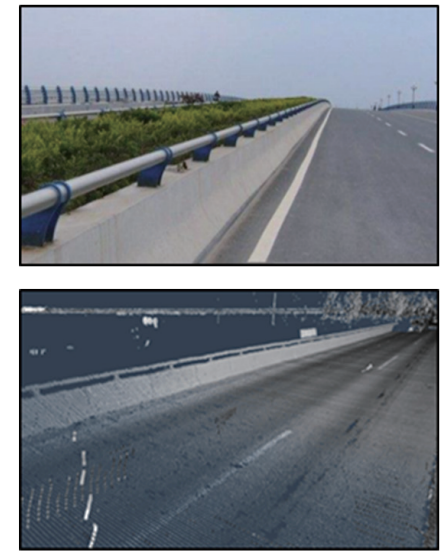

(b)
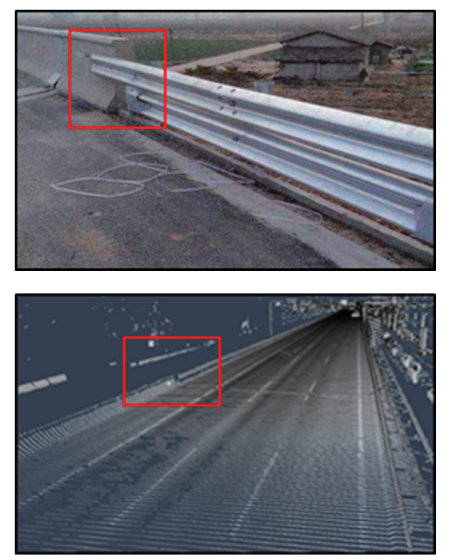

(c)

Fig. 1. (Color online) Two main types of guardrail on highways. (a) Steel guardrail, (b) concrete guardrail, and (c) connection between the two types of guardrail.

In the existing literature on highway target recognition, there has been little research on guardrail extraction, and most of the extraction methods focus on the recognition of guardrail targets from images. ${ }^{(1)}$ In order to improve and accelerate vehicle detection algorithms, Broggi and coworkers ${ }^{(2,3)}$ studied the method of guardrail extraction. A laser point cloud was transformed into a binary image, and then the edge was analyzed. According to the length of the edge, they determined whether the object was a guardrail. This method was only applicable to a driving speed of less than $5 \mathrm{~km} / \mathrm{h}$ and has low applicability. In 2013, Seibert et $a l .{ }^{(4)}$ used an image processing method to detect the soft edges, curbs, and guardrails on both sides of a road. Firstly, a neural network was used to detect the boundaries of roads, i.e., road edges, and then the Lucas-Kanade algorithm ${ }^{(5)}$ was used to track Harris ${ }^{(6)}$ features, and 3D information was extracted from them to complete the identification of guardrails. Owing to the image-based method, the specific position of the guardrail could not be determined, and the recognition accuracy was low. A LIDAR point cloud has the characteristics of high precision, and the problem of low image accuracy can be effectively solved by directly identifying the guardrail point cloud from the laser point cloud. Using the scan line information of a vehicle point cloud, Peng ${ }^{(7)}$ extracted guardrails from the abrupt change between the guardrail points and the road points. However, the method was overreliant on the scan line information and easily affected by noise points. Jiang et al. ${ }^{(8)}$ also segmented a single scan line to extract candidate guardrail point clouds. In addition, the method estimated the guardrail feature points from the candidate guardrail point clouds. Finally, a guardrail tracking algorithm was proposed to refine the guardrail feature points and obtain the final detection results. Guo ${ }^{(9)}$ proposed a detection method for expressway guardrails based on distance and the filtering of redundant feature points. The method first divided the region of interest, and then extracted the guardrail according to the height and angle features of the scanning line of the guardrail. This method could effectively extract a corrugated guardrail but could not identify a concrete guardrail. 
In the existing image-based recognition methods, the recognition accuracy of guardrails is generally low. Most of the methods based on a laser point cloud are overreliant on the scanning line information and only recognize a small amount of real-time point cloud data, and they cannot effectively distinguish concrete and steel guardrails on the highway. To overcome the above defects, in this paper, we apply an improved efficient binary ecoding voxel plane segmentation algorithm (EVBS) based on the voxel segmentation of a point cloud ${ }^{(10)}$ to filter out the pavement and reduce the data volume, and then a voxel Euclidean clustering point cloud segmentation method is proposed to improve the efficiency of clustering. The slicing method combined with the geometric space characteristics of the guardrail is used to extract the guardrail from the clustered target. Finally, a method based on the characteristics of the guardrail cross section is proposed to distinguish steel and concrete guardrails in an expressway.

\section{Method}

\subsection{Pavement removal}

In the vehicle laser point cloud, the pavement point cloud accounts for the vast majority of the data. Filtering the pavement in the road scene can reduce the amount of data and improve the efficiency and accuracy of guardrail identification. This paper employs an EVBS with improved efficiency ${ }^{(10)}$ to segment the pavement: we change the seed selection strategy and regional growing conditions to adapt to the pavement segmentation. Firstly, the voxel height, number of points, and flatness are used as seed selection factors. According to the characteristics of the pavement point cloud, the region growth conditions set in the voxel segmentation process of the algorithm are as follows:

$$
\left\{\begin{array}{c}
\left|z_{S}-z_{N}\right|<\sigma_{h} \\
r_{N}<\sigma_{r} \\
\overrightarrow{n_{S}} \cdot \overrightarrow{n_{N}}<\sigma_{\theta}
\end{array}\right.
$$

where $S$ is the seed voxel, $N$ is the adjacent voxel of the seed voxel, $\sigma$ is the threshold, $r$ is the residual, $n$ is the normal, and $\theta$ is the angle between the seed and the adjacent voxel. If the adjacent voxels and the seed voxel meet the above three conditions, they are classified as pavement voxels. During the voxel division of the EVBS, there will be point clouds with two or more targets in the same voxel, as shown in Fig. 2(b), at the edge of the pavement; the voxels in the red box contain two types of point cloud: pavement and guardrail. Because the residual value and elevation of this type of voxel are large, it will not be classified into pavement in the process of regional growth. As shown in Fig. 2(c), the pavement edge extraction is not accurate. In this paper, according to the distance relationship between the undivided voxels and the segmented pavement voxels, the pavement point clouds in the multi-objective voxels are refined and segmented. If the distance between the point cloud in the voxel adjacent to the pavement satisfies $d_{i}<\sigma_{d}$, the point is considered as a pavement point, as shown in Fig. 2(d). 

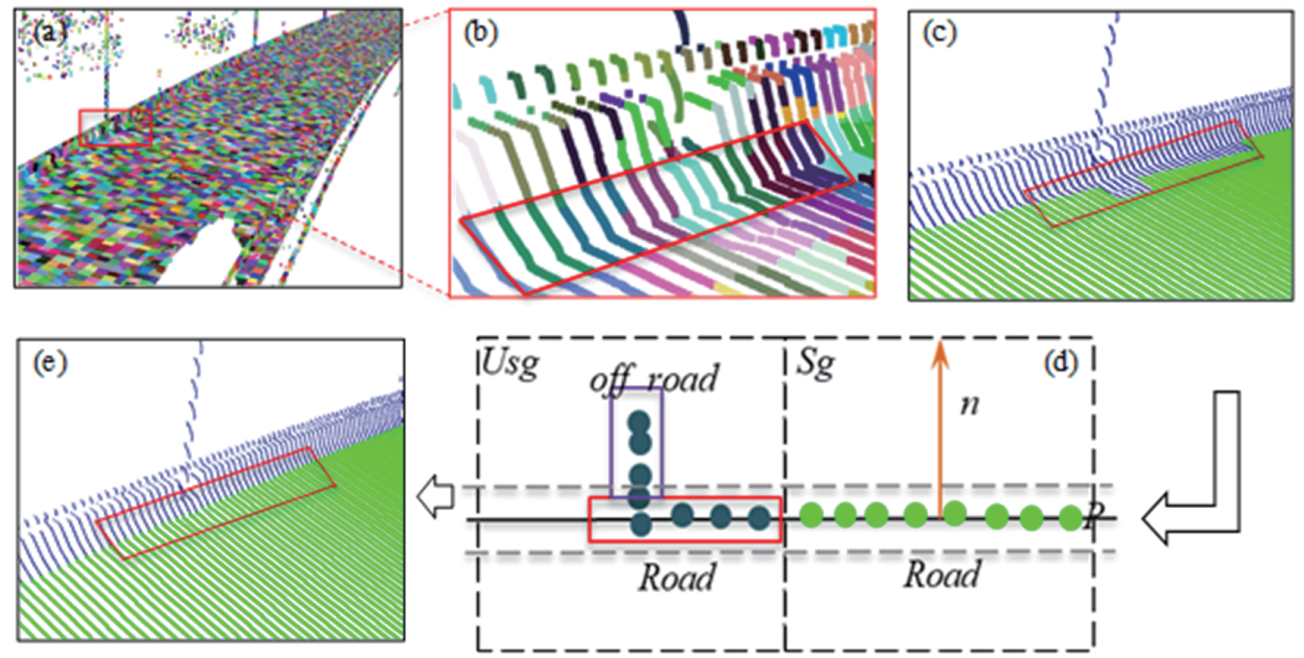

Fig. 2. (Color online) Point cloud subdivision of unsegmented voxel. (a) Voxelization of point cloud, (b) multiobjective voxels in pavement edge, (c) poor pavement edge segmentation effect, (d) subdivision of undivided pavement points, and (e) better pavement edge segmentation effect.

\subsection{Extraction of point cloud of guardrail}

The pavement extraction method in Sect. 2.1 is used to remove the pavement in the point cloud, and most of the objects in the point cloud scene are separated from each other, as shown in Fig. 3. It can be seen that the guardrails on both sides of the road are continuous, but the guardrail is connected with the street lamp or the low platform below, so it is necessary to distinguish them in the extraction.

\subsubsection{Voxelization clustering segmentation of off-ground points}

After the ground points are filtered out, the off-ground points remain separate and unorganized. Before the guardrail extraction, the points belonging to the same cluster must be further grouped. The traditional Euclidean clustering method for point cloud needs to use a K-D tree to organize the point cloud, and needs to query the neighborhood points of each point, then gather the point clouds belonging to the same region in space into a cluster. To further improve the efficiency of point cloud clustering, we propose a voxel Euclidean clustering point cloud segmentation method to improve the segmentation efficiency.

Voxelization of point clouds. First, we set the voxel size $d_{I}$, and then use the efficient binary ecoding method ${ }^{(10)}$ to construct the octree for the point cloud and complete the voxelization of the off-ground point cloud, as shown in Fig. 3(a).

Selection of seed voxel. In this paper, the number of points in voxel $v_{n}$ is used as the standard for seed voxel selection, and the voxels are selected in order of decreasing value.

Voxel growth conditions. In the process of voxel region growth, it is necessary to compare the similarity between seed voxels and their adjacent voxels, and then merge the similar voxels. 


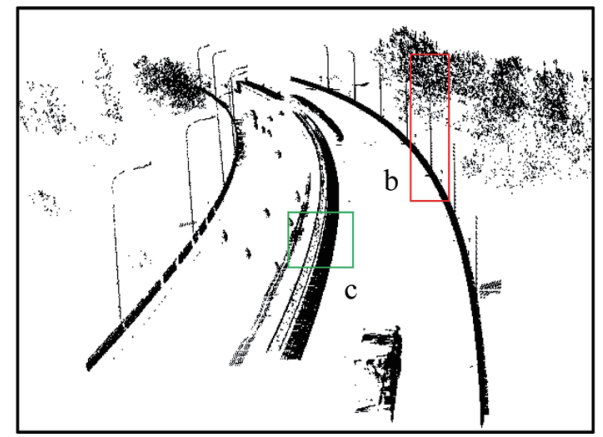

(a)

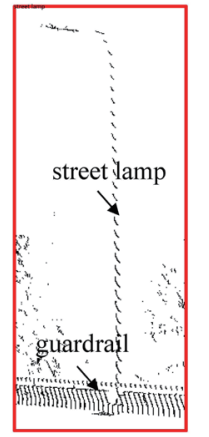

(b)

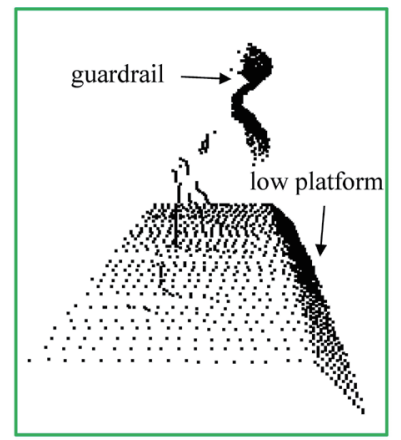

(c)

Fig. 3. (Color online) Off-ground points after ground removal. (a) Off-ground points after ground removal, (b) guardrail connected with the street lamp, and (c) guardrail connected with the low platform.

In this process, spatial distance similarity is the most important similarity feature. Another feature is that the point cloud in the two voxels should have continuity, which is expressed as follows:

$$
\left\{\begin{array}{c}
D_{i j}=\left|\overline{X_{i}}-\overline{X_{j}}\right| \\
D_{i j}<d_{I} / 2
\end{array},\right.
$$

where $D_{i j}$ is the Euclidean distance of the mean value $\overline{X_{i}}, \overline{X_{j}}$ of the point cloud coordinates between the seed voxel and the adjacent voxels, as shown in Fig. 3(b).

Voxel region growth. By the above method of selecting seed voxels and the above growth conditions, the voxel region growth algorithm is used to complete the clustering segmentation of off-the-ground point cloud, as shown in Figs. 3(c) and 4(d).

\subsubsection{Slice method to extract guardrail}

After voxel clustering, each cluster presents certain characteristics in space. The guardrail has the following characteristics: (1) the guardrail is located on both sides of the road. After spatial clustering, the point cloud of a steel guardrail is usually connected with a low platform with grass below. A concrete guardrail is relatively independent, but it is sometimes connected with street lamps; (2) the guardrail is usually continuous and has a large length; (3) the height of the guardrail is relatively fixed; (4) some of the ground objects behind a steel guardrail are covered, while the ground features behind the concrete guardrail are completely covered.

On the basis of the above characteristics, we use a slice method ${ }^{(11)}$ to extract the guardrail from the clustered targets. Firstly, the bounding box of a single point cloud is calculated, and then the point cloud is sliced along the $x$-axis or $y$-axis. The selection of the coordinate axis is determined by the following formula: 

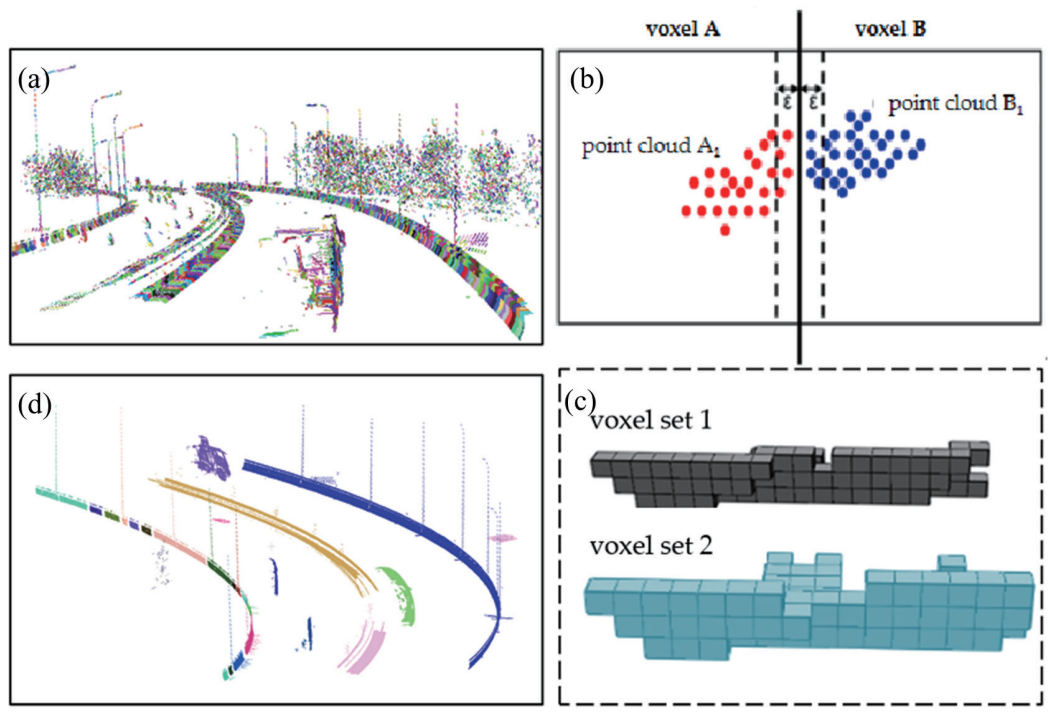

Fig. 4. (Color online) Voxelization clustering and segmentation process of off-ground points. (a) Off-ground point voxelization, (b) voxelization Euclidean clustering conditions, (c) voxel clusters after clustering, and (d) object point cloud after spatial clustering.

$$
\text { delt }=\left\{\begin{array}{c}
\Delta x=x_{\max }-x_{\min } \\
\Delta y=y_{\max }-y_{\min } \\
\Delta x>\Delta y ? \Delta x: \Delta y
\end{array} .\right.
$$

After the coordinate axis of the slicing is determined, the coordinate value difference delt is also calculated. Then the width $s$ of the slice and the number of slices $m=d e l t / s+1$ are set. Taking the $x$-axis slice as an example, the coordinates of the point cloud on a piece are as follows:

$$
\left\{\begin{array}{c}
x \in\left[x_{\min }+i \cdot s, x_{\min }+(i+1) \cdot s\right] i=0,1,2, \ldots, m \\
y, \quad z \in R
\end{array}\right.
$$

The experimental results of this study show that $s=1.5 \mathrm{~m}$ can obtain a good extraction effect. Figure 5 shows the rules of slicing and the results of guardrail slicing. Each slice is represented in a different color.

After slicing each cluster point cloud, the height of the slice can be calculated from the $z$-coordinate difference of each point cloud. From our prior knowledge of guardrails, the height of a guardrail is usually between 0.5 and $1.2 \mathrm{~m}$, and the guardrail is longer and the number of slices is more than other off-ground objects. The number of guardrails that meet the height threshold $n$ and the total number of slices $N$ of each guardrail slice are counted. The slice height and statistical threshold are calculated as follows: 

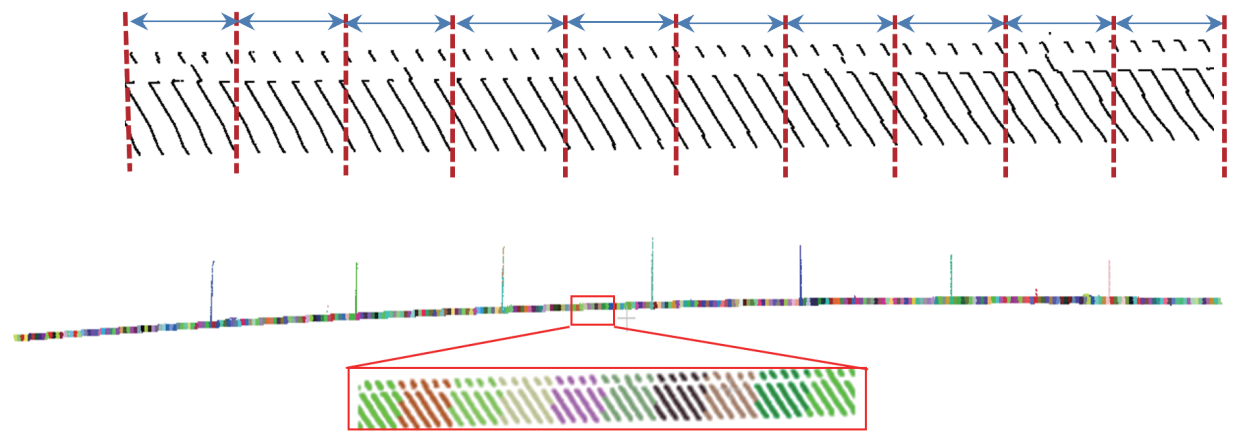

Fig. 5. (Color online) Schematic diagram of guardrail slicing.

$$
\left\{\begin{array}{l}
h_{c l i p[i]}=z_{\max }-z_{\min } \\
h_{i} \in\left\{H \mid \lambda_{1}<h_{i}<\lambda_{2}\right\}
\end{array} .\right.
$$

If $n / N>60 \%$ and $n>10$, then the cluster is considered as a guardrail.

After a cluster is identified as a guardrail, two types of non-guardrail point clouds remain. One corresponds to guardrail slices connected with street lamps, as shown in Fig. 3(b), and the other corresponds to the low platform under the steel guardrail, as shown in Fig. 3(c). In this paper, after the classification of the guardrail type (Sect. 2.3), the low platform is removed by considering the height of the guardrail. Firstly, the range of height values of all slices of a certain section of guardrail is divided into a series of intervals, and then the statistical histogram of the height values of the guardrail is calculated. Finally, the average value of the height $h_{g}$ that falls in the highest frequency interval of the histogram is taken as the height of the guardrail:

$$
h_{g}=\frac{1}{m} \sum_{i=0}^{m} h_{i}
$$

For the first case, points with $Z$ coordinate higher than the overall height of the guardrail, $z_{p_{i}}>h$, are removed. For the second case, according to the design rules of the steel guardrail, the height of the steel plate $h_{g_{s}}$ is not more than $500 \mathrm{~mm}$. Therefore, the points with $z_{p_{i}}<z_{\text {max }}-h_{g_{s}}$ in the section of the guardrail are removed since they are from the low platform.

\subsection{Distinction between steel and concrete guardrails}

By considering the height of the slice, the above method can extract the guardrail without distinguishing its type. However, there are two main types of guardrail: steel guardrails and concrete guardrails, which need to be distinguished in practical applications such as road inspection. Because these two types of guardrail are similar in height and are connected adjacently [Fig. 1(c)], it is difficult to distinguish them by height and position. Through research 
and comparison, we found that the two types of guardrail have obvious differences in their cross section. The section of the steel guardrail is corrugated and usually connected with the lower platform, whereas the concrete guardrail has a zigzag structure, as shown in Fig. 6. Using this feature, we propose an interior point statistical method by fitting a plane based on section features to distinguish the two types.

In this part, the section characteristics of a guardrail are analyzed by using the guardrail slices discussed in Sect. 2.2. For each slice, the center point $O(X, Y, Z)$ of the guardrail point cloud is calculated first. Then, the outer product of the eigenvector corresponding to the maximum eigenvalue of the covariance matrix ${ }^{(12)} \vec{e}_{3}$ of the point cloud and the positive vector $\vec{z}$ of the $Z$ coordinate axis, $\vec{n}=\vec{e}_{3} \times \vec{z}$, is taken as the normal vector of the plane to obtain the fitting plane equation of the guardrail. This plane is parallel to the guardrail and perpendicular to the ground, as shown in Fig. 6 . Then the distance between each point $p_{i}\left(x_{i}, y_{i}, z_{i}\right)$ and the plane is calculated. Next, a threshold distance $d$ is set: if $d_{i}<d$, the point is considered as an interior point of the plane. It can be seen from Fig. 6 that there are more interior points for the concrete guardrail than for the steel guardrail. If the proportion of internal points is greater than a threshold $\sigma_{n}$, the point cloud of the slice is considered to be a concrete guardrail; otherwise, it is a steel guardrail.

\section{Experiments and Analysis}

\subsection{Data description}

The road survey area in our study comprised sections of the Beijing Kaifeng highway of Daxing District, Beijing, as shown in Fig. 7. During the investigation, the driving speed was about $60 \mathrm{~km} / \mathrm{h}$, the distance between points on the scanning line was about $14 \mathrm{~mm}$, the distance between scanning lines was about $5 \mathrm{~cm}$, and the total length of scanning was $22.974 \mathrm{~km}$. After

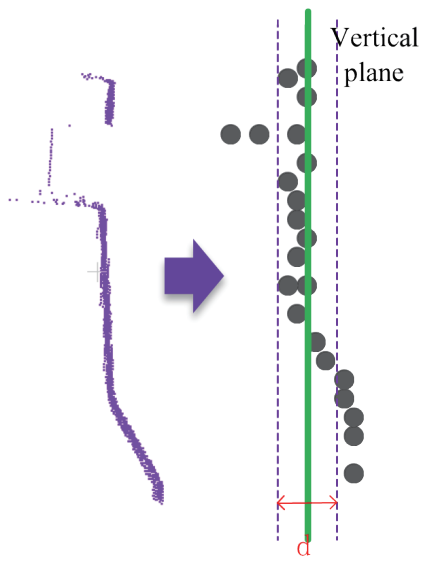

(a)

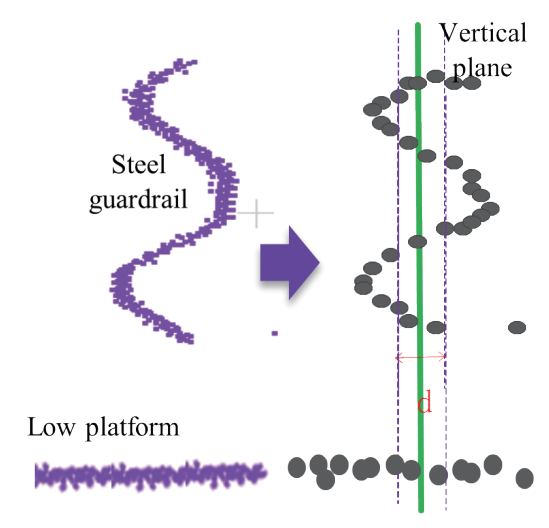

(b)

Fig. 6. (Color online) Cross sections of two types of guardrail. (a) Concrete guardrail model, (b) steel guardrail model. 

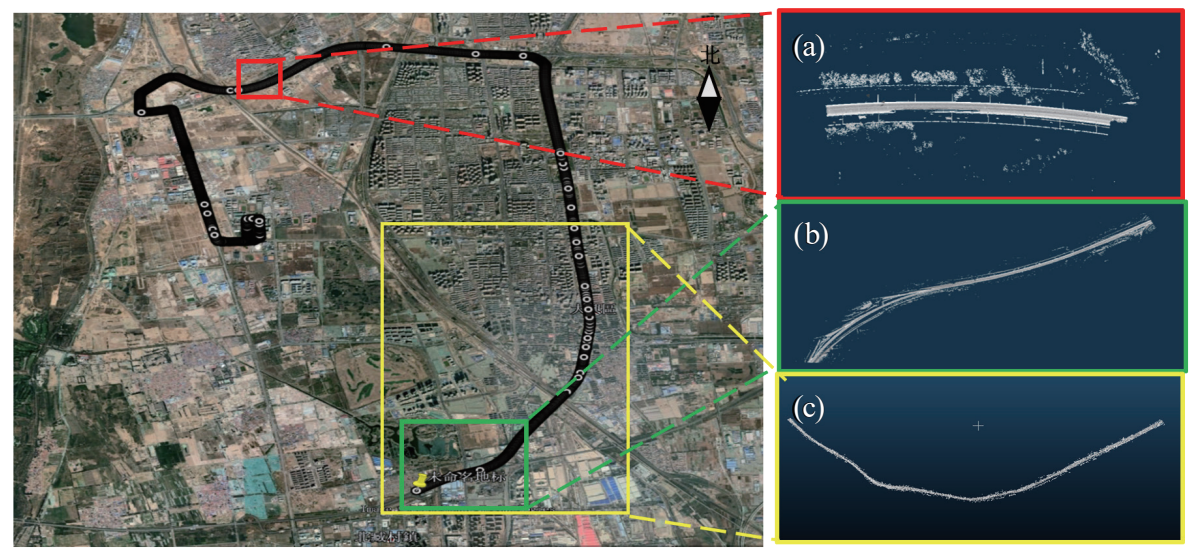

Fig. 7. (Color online) Sections of research road. (a) First group of data, (b) second group of data, and (c) third group of data.

calculation and calibration, the relative accuracy of the data was higher than $4 \mathrm{~mm}$ and the absolute accuracy (plane/elevation) was higher than $40 \mathrm{~mm} / 60 \mathrm{~mm}$.

The first group of data was taken from a suburban expressway. The mobile laser scanning system ran slowly and the point cloud density was high. There are street lamps distributed on both sides of the road. There are also many miscellaneous points such as trees and weeds on both sides of the road. The number of point clouds in this group is 3576043 and the length of the section is $323 \mathrm{~m}$, as shown in Fig. 7(a). The second group of point clouds was taken from a highway section far from the urban area. Owing to the high driving speed, the point cloud is relatively sparse, and there are few trees or street lights on both sides. The number of points is 20059733 and the length is $1.667 \mathrm{~km}$, as shown in Fig. 7(b). The third group of data is an extended section incorporating the second group of data. The amount of point cloud data in this section is about 120 million and the length is about $8 \mathrm{~km}$. Because of the length of the road section, the scene is more complex, and there are many types of miscellaneous points, as shown in Fig. 7(c).

\subsection{Accuracy evaluation}

To evaluate the effectiveness and accuracy of the guardrail extraction algorithm proposed in this paper, the manual segmentation method is used for the three selected groups of point cloud data to segment and classify the ground objects on the road, and the results of manual segmentation are taken as the ground truth. The segmentation algorithm is evaluated by comparing the results of automatic segmentation with those of manual segmentation. Three metrics, precision, recall, and comprehensive quality $\mathrm{F} 1,{ }^{(13-16)}$ are selected as evaluation criteria.

$$
\text { precision }=\frac{|T P|}{|T P|+|F P|}
$$




$$
\begin{gathered}
\text { recall }=\frac{|T P|}{|T P|+|F N|} \\
F 1=2 \times \frac{\text { precision } \times \text { recall }}{\text { precision }+ \text { recall }}
\end{gathered}
$$

True positive (TP): number of $p_{t}, p_{t} \in\left(s \cap s^{\prime}\right)$; (2) false positive (FP): number of $p_{f}, p_{f} \in s^{\prime}, p_{f} \notin s$; (3) false negative (FN): number of $p_{n}, p_{n} \in s, p_{n} \notin s^{\prime}$.

\subsection{Experimental results}

Figure 8 shows the results of guardrail extraction from the three groups of point cloud data. To better express the extraction results and the positions of guardrails, the extracted pavement point cloud is represented in red, the extracted concrete guardrail is represented in green, and the steel guardrail is represented in light blue. It can be seen from the figure that the proposed method can extract the point cloud of the guardrail effectively, and can accurately distinguish the concrete and steel guardrails. The extracted point cloud of the concrete guardrail can be accurately separated from the connected ground point cloud, and the boundary is clear and complete. For the steel guardrail, the corrugated steel plate point cloud is correctly identified with good integrity. In addition, the proposed method is also effective in abnormal situations, such as the intersection of the two types of guardrail, and double corrugated guardrails.

Owing to the lack of existing guardrail extraction algorithms and the fact that most of them are for image data recognition, to evaluate the performance of our proposed algorithm, we use
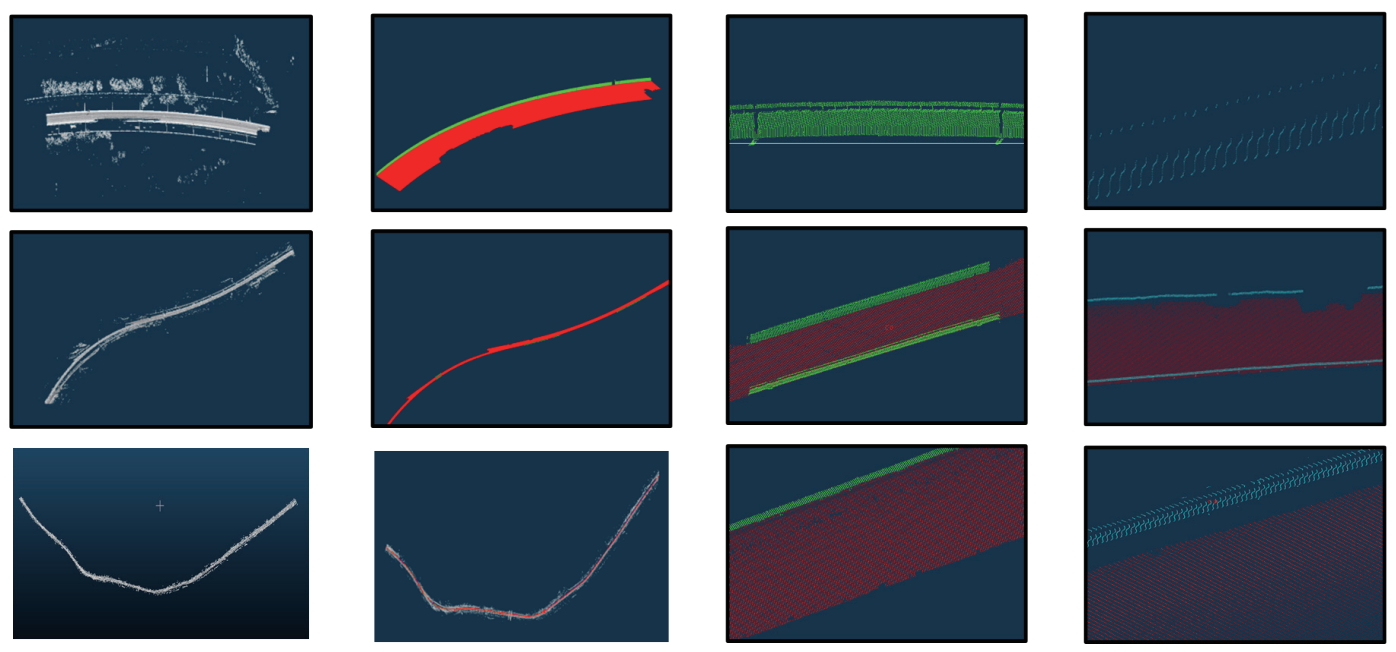

(a)

(b)

(c)

(d)

Fig. 8. (Color online) Extraction results of guardrail point cloud of three groups of point cloud data. (a) Original data, (b) overall recognition results, (c) details of concrete guardrail recognition, and (d) details of steel guardrail recognition. 
a guardrail extraction method based on scan line information ${ }^{(7)}$ as an algorithm for comparison, which is easy to implement. To evaluate the extraction quality of the proposed guardrail extraction algorithm, the TP, FP, and FN values of the two algorithms for guardrail extraction were counted. The evaluation results are shown in Table 1.

In the scan-line-based method, each scanning line is taken as the study object, and the number of points in the range of radius $R$ is counted. The scanning point with the largest number of points in the range is taken as the center point of the guardrail, and a point whose peripheral height is greater than $\sigma_{h}$ is taken as a guardrail point. Therefore, the influence of street lamps and weeds leads to a selection error in the center point and an incorrect segmentation result. It can be seen from Table 1 that the three evaluation metrics of the guardrail point cloud extracted by this method are all below 0.9. The proposed method first clusters the point clouds, and then extracts them by the slice method according to the position characteristics and morphological characteristics of the guardrail. The comprehensive extraction quality F1 and recall rate of the first and second groups of data are both 0.97 and 0.95 , respectively. Owing to the large number of point clouds in the third group of data and the more complex scene, the extraction accuracy is slightly reduced to 0.92 and F1 is 0.91 , which is still significantly better than the value of 0.76 for the scan-line-based method. In terms of the recognition time, the proposed algorithm is clearly advantageous, taking only $35.3 \mathrm{~s}$ to identify the guardrail for data 3.

Since our method distinguishes concrete and steel guardrails, it is necessary to evaluate the identification quality for these two types of guardrail. As shown in Table 2, it can be seen that the precision of concrete guardrail identification is relatively high, with values of $0.98,0.96$, and 0.93 for the three groups of data. This is because the point cloud characteristics of the concrete guardrail are relatively obvious: most of the point clouds of their slice are planar and the height is relatively stable. In the identification of the steel guardrail, the height of slices is unstable owing to the uneven ground below it, which easily causes incorrect segmentation. Therefore, the accuracy of steel guardrail identification is slightly lower, with values of $0.94,0.89$, and 0.84 for the three groups of data.

Table 1

Accuracy of guardrail extraction.

\begin{tabular}{|c|c|c|c|c|c|c|c|c|}
\hline \multirow{2}{*}{ Data } & \multicolumn{4}{|c|}{ Guardrail extraction based on scan line } & \multicolumn{4}{|c|}{ Proposed method } \\
\hline & Precision & Recall & $\mathrm{F} 1$ & Time/s & Precision & Recall & F1 & Time/s \\
\hline Data 1 & 0.87 & 0.83 & 0.85 & 7.6 & 0.97 & 0.97 & 0.97 & 0.35 \\
\hline Data 2 & 0.81 & 0.79 & 0.80 & 56.7 & 0.96 & 0.95 & 0.95 & 3.4 \\
\hline Data 3 & 0.75 & 0.78 & 0.76 & 203.5 & 0.92 & 0.90 & 0.91 & 35.3 \\
\hline
\end{tabular}

Table 2

Accuracy of extraction of two types of guardrail.

\begin{tabular}{lccccccc}
\hline \multirow{2}{*}{ Data } & \multicolumn{3}{c}{ Concrete guardrail } & & \multicolumn{3}{c}{ Steel guardrail } \\
\cline { 2 - 4 } \cline { 6 - 8 } & Precision & Recall & F1 & & Precision & Recall & F1 \\
\hline Data 1 & 0.98 & 0.97 & 0.97 & & 0.94 & 0.94 & 0.94 \\
Data 2 & 0.96 & 0.96 & 0.96 & & 0.89 & 0.87 & 0.88 \\
Data 3 & 0.93 & 0.91 & 0.92 & & 0.84 & 0.85 & 0.84 \\
\hline
\end{tabular}




\section{Conclusion}

Guardrails are important ancillary facilities on the highway, which not only provide road edge information but also play a role in protecting drivers. However, the existing research on highway guardrail extraction and recognition is insufficient and cannot accurately and efficiently extract and distinguish guardrails and their type. In view of the above defects, we propose a clustering slicing method to identify the guardrails in a road scene from their characteristics. Before the identification of the guardrail target, an improved binary coded octree plane segmentation algorithm is applied to pavement point filtering, which reduces the amount of data in the guardrail identification and improves the recognition efficiency. Then a voxel Euclidean clustering point cloud segmentation method is proposed to improve the efficiency of target clustering. Employing the geometric and spatial characteristics of the guardrail, a slice method is used to accurately identify the guardrail after clustering. A method to distinguish steel and concrete guardrails based on their cross-section characteristics is also proposed. The experimental results show that the proposed method can effectively and accurately identify the guardrail in the point cloud scene of the expressway, and the overall recognition accuracy is more than $90 \%$. It can also effectively distinguish the type of guardrail with greater accuracy and in a shorter time than in other studies. Moreover, the algorithm has obvious advantages in terms of efficiency for automating the process for large sets of point clouds.

However, in some parts of the expressway data, the guardrail is interrupted owing to vehicle occlusion. In this case, the guardrail is identified as multiple guardrails. In future work, connectivity analysis will be carried out to resolve this problem. In addition, because of the recognition based on the geometric and spatial characteristics of the guardrail, some thresholds need to be set on the basis of prior knowledge. How to reduce the use of thresholds will be a focus of future research.

\section{Acknowledgments}

This research was funded by the Fundamental Research Funds for Beijing University of Civil Engineering and Architecture (grant no.X20088), the National Key Research and Development Program of China (grant no. 2016YFC0802107), the National Natural Science Foundation of China (grant nos. 41971350 and 41871367), the Importation and Development of HighCaliber Talents Project of Beijing Municipal Institutions (grant no. CIT\&TCD201704053), the Science and Technology Project of Ministry of Housing and Urban-Rural Development of the People's Republic of China (grant no. 2017-K4-002), the Scientific Research Project of Beijing Educational Committee (grant no. KM201910016005), the Major Projects of Beijing Advanced Innovation Center for Future Urban Design (grant no. UDC2018031321), the Talent Program of Beijing University of Civil Engineering and Remote Architecture, the Fundamental Research Funds for Central and Beijing Universities (grant nos. X18051 and X18014), and the BUCEA Postgraduate Innovation Project (grant no. PG2020073). 


\section{References}

1 H. Zhu, X. Wang, and B. Guo: 2018 Chinese Automation Congress (CAC) (IEEE, 2018) 777-782.

2 Broggi, P. Cerri, F. Oleari, and M. Paterlini: IEEE Intelligent Transportation Systems (IEEE, 2005) 552-556.

3 G. Alessandretti, A. Broggi, and P. Cerri: Trans. Intell. Transp. Syst. 8 (2007) 95. https://doi.org/10.1109/ TITS.2006.888597

4 A. Seibert, M. Hähnel, A. Tewes, and R. Rojas: 2013 IEEE Intelligent Vehicles Symposium (IV) (IEEE, 2013) 853-858.

5 B. D. Lucas and T. Kanade: Proc. Ijcai. 81 (1981) 674. https://ri.cmu.edu/pub_files/pub3/lucas_bruce_ d_1981_2/lucas_bruce_d_1981_2.pdf

6 C. Harris and $\bar{M}$. Stephens: Proc. 4th Alvey Vision Conf. 15 (1988) 10. http://citeseerx.ist.psu.edu/viewdoc/ download?doi $=10.1 \cdot 1.434 .4816 \&$ rep $=$ rep1\&type $=$ pdf

7 J. F. Peng: Study on automated extraction methods of highway elements from mobile Lidar point clouds. Beijing University of Civil Engineering and Architecture (2017). https://kns.cnki.net/KCMS/detail/detail. aspx?dbname $=$ CMFD201801\&filename $=1017169520 . \mathrm{nh}$

8 Y. Jiang, B. He, L. Liu, R. Ai, and X. P. Lang: 2016 IEEE 19th Int. Conf. Intelligent Transportation Systems (ITSC) (IEEE, 2016) 1540-1545

9 B. Guo: Study on Highway Guardrail Detection Method Using Lidar. Chongqing University of Posts and Telecommunications (China National Knowledge Infrastructure, 2019). https://kns.cnki.net/KCMS/detail/ detail.aspx?dbname $=$ CMFD202001\&filename $=1019643582 . \mathrm{nh}$

10 M. Huang, P. C. Wei, and X. L. Liu: Remote Sens. 11 (2019) 2727. https://doi.org/10.3390/rs11232727

11 S. M. I. Zolanvari and D. F. Laefer: ISPRS J. Photogramm. Remote Sens. 119 (2016) 334. https://doi. org/10.1016/j.isprsjprs.2016.06.011

12 A. Nurunnabi, D. Belton, and G. West: 2012 Int. Conf. Digital Image Computing Techniques and Applications (DICTA, 2012) 1-8.

13 Z. Sun, Y. Xu, L. Hoegner, and U. Stilla: ISPRS Annals of Photogrammetry, Remote Sensing \& Spatial Information Sciences 4 (2018) 271. https://doi.org/10.5194/isprs-annals-IV-2-271-2018

14 Z. Cai, H. Ma, and L. Zhang: Remote Sens. 11 (2019) 848.

15 A. V. Vo, L. Truong-Hong, and D. F. Laefer: Remote Sens. 104 (2015) 88.

16 R. Schnabel, R. Wahl, and R. Klein: Comput. Graph. Forum 26 (2007) 214.

\section{About the Authors}

Ming Huang is a professor at Beijing University of Civil Engineering and Architecture. His research interests are in point cloud \& image hyperfine 3D modeling and visualization, and mobile vehicle laser point cloud ground feature intelligent recognition.

(huangming@bucea.edu.cn)

Chuanli Zhou is a graduate student of Beijing University of Civil Engineering and Architecture. His research interests are in 3D laser scanning, 3D modeling, and visualization. (2108160219009@stu.bucea.edu.cn)

Pengpeng Huo received her B.S. degree from China University of Geosciences (Wuhan), China, in 2016. She has been studying for her M.S. at Beijing University of Civil Engineering and Architecture, China, since 2018. Since 2016, she has been a surveying and mapping engineer at the Beijing Institute of Surveying and Mapping, China. Her research interests are in remote sensing, LiDAR point clouds, and 3D reconstruction.

(2208521518001@stu.bucea.edu.cn)

Zhiqun Wu is general manager of Beijing Beijianda Technology Co., Ltd. His research interests are in 3D laser scanning and digital protection of architectural heritage.

(wuzhiqun@bucea.edu.cn) 